\title{
Inserting two atoms into a single optical micropotential
}

\author{
Y. Miroshnychenko, W. Alt, I. Dotsenko, L. Förster, \\ M. Khudaverdyan, D. Meschede, S. Reick, and A. Rauschenbeute* \\ Institut für Angewandte Physik, Universität Bonn, Wegelerstr. 8, D-53115 Bonn, Germany
}

(Dated: June 18, 2021)

\begin{abstract}
We recently demonstrated that strings of trapped atoms inside a standing wave optical dipole trap can be rearranged using optical tweezers [Y. Miroshnychenko et al., Nature, in press (2006)]. This technique allows us to actively set the interatomic separations on the scale of the individual trapping potential wells. Here, we use such a distance-control operation to insert two atoms into the same potential well. The detected success rate of this manipulation is $16_{-3}^{+4} \%$, in agreement with the predictions of a theoretical model based on our independently determined experimental parameters.
\end{abstract}

PACS numbers: 32.80.Lg, 32.80.Pj, 39.25.+k, 03.67.-a

Controlled interaction between pairs of neutral atoms in optical micropotentials leads to a number of interesting applications ranging from the highly efficient production of ultracold molecules [1, 2, 3, 4, 5, 6] to the coherent interaction of atoms through controlled cold collisions. Such collisions have been shown to yield state-dependent collisional phase shifts [7] and to lead to coherent spin dynamics [8]. Both effects are candidates for creating entanglement and for realizing coherent conditional dynamics, of great relevance in quantum information processing (QIP).

So far, these experiments were carried out with large samples of ultracold or quantum degenerate atoms, transferred into the motional ground state of optical lattices [9]. In combination with the high atomic densities, this results in excellent starting conditions for the above schemes, albeit at the expense of the lack of addressability at the single atom level. While ensemble measurements still yield information about processes like, e.g., the entangling and disentangling dynamics 7], their use for those QIP applications requiring the measurement of individual quantum states is impaired.

In our "bottom-up" approach, on the other hand, neutral atom systems are built atom-by-atom while maintaining full control over the degrees of freedom of each individual atom. When stored in a standing wave dipole trap, formed by a pair of counterpropagating laser beams, the absolute positions of individual atoms along the beam axis can be optically measured with sub-micrometer precision and the number of potential wells separating simultaneously trapped atoms can be exactly determined [10]. In addition, we have demonstrated that the quantum state of individual atoms in the standing wave dipole trap can be selectively prepared and read out with a high spatial resolution [11]. Furthermore, the atoms can be positioned along the trap axis using the dipole trap as an "optical conveyor belt" 10, 12]. Finally, using optical tweezers, we have recently rearranged the so far irregu-

*Electronic address: rauschenbeutel@iap.uni-bonn.de

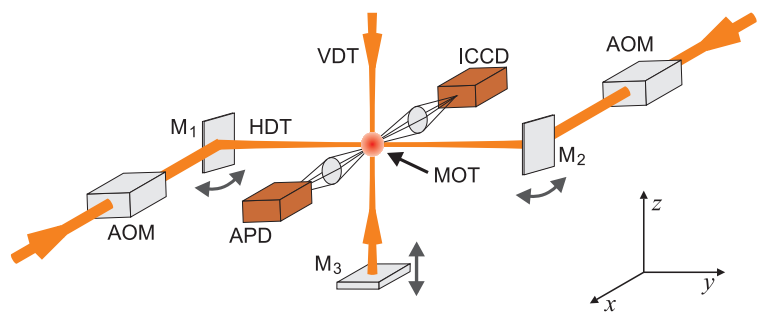

FIG. 1: (Color online) Scheme of our experimental setup. Two crossed standing wave dipole traps are used to rearrange pairs of trapped neutral atoms. See text for details.

larly spaced atoms into regularly spaced strings [13].

Here, we present first results concerning the insertion and controlled interaction of two individual atoms inside the same optical micropotential. The atoms are initially stored in separate potential wells of a standing wave dipole trap. One of the two atoms is then extracted out of its potential well using optical tweezers and inserted into the potential well of the second atom.

In the rearrangement experiment [13], the final interatomic distances were verified by recording fluorescence images. When the two atoms ideally end up confined in a volume of the order of one cubic optical wavelength, however, they cannot be optically resolved. The successful insertion of the two atoms into one potential well is therefore detected by irradiating the atoms with near resonant light, inducing inelastic collisions. These collisions lead to a loss of the atoms and occur if and only if the atoms occupy the same potential well.

The essential parts of our setup are schematically depicted in Fig. 1] A horizontal standing wave dipole trap (HDT) is formed by two counterpropagating Nd:YAG laser beams with a wavelength of $\lambda_{\mathrm{HDT}}=1064 \mathrm{~nm}$ and a power of $1 \mathrm{~W}$ each. They are focused to a waist of $w_{\mathrm{HDT}}=19 \mu \mathrm{m}\left(1 / e^{2}\right.$-radius $)$, generating a chain of potential wells, separated by $\lambda_{\mathrm{HDT}} / 2=532 \mathrm{~nm}$ with a measured depth of $U_{\mathrm{HDT}} / k_{\mathrm{B}}=0.8 \mathrm{mK}$. The HDT is loaded with an exactly known number of caesium atoms from a high gradient magneto-optical trap (MOT), as inferred from the discrete MOT fluorescence levels, recorded with 
an avalanche photodiode (APD). The single atom transfer efficiency between the traps is $98.7_{-1.1}^{+0.7} \%$. Following the transfer from the MOT, we let the atoms freely expand along the DT axis by switching off one of the beams for $1 \mathrm{~ms}$. The atoms are then randomly distributed over an interval of about $80 \mu \mathrm{m}$ along the axis of the trap. Subsequently, we record a fluorescence image using an intensified CCD camera (ICCD) 14]. For this purpose, we illuminate the atoms with a near resonant three-dimensional optical molasses, thereby also cooling the atoms to a temperature of about $80 \mu \mathrm{K}$. From the ICCD image with $1 \mathrm{~s}$ exposure time, the position of all optically resolved atoms are determined with an uncertainty of $\Delta y_{\text {position }}=140 \mathrm{~nm}$ rms along the axis of the HDT, significantly smaller than the $532 \mathrm{~nm}$ separation between adjacent potential wells of the HDT [10].

The atoms in the HDT can be moved along the $x$ and $y$-directions, see Fig. 1 Transport along the HDT axis, i.e., the $y$-direction, is achieved by means of our "optical conveyor belt" method [12, 15]. For this purpose, acousto-optic modulators (AOM) mutually detune the laser frequencies. The moving standing wave pattern thus transports the atoms over distances of up to a few millimeters with submicrometer precision [10] within a few hundred microseconds.

Displacing the HDT in the $x$-direction, i.e., perpendicular to its axis, is realized by synchronously tilting the mirrors $\mathrm{M}_{1}$ and $\mathrm{M}_{2}$ in opposite directions about the $z$ axis using PZT actuators. For small tilts of $\sim 0.1 \mathrm{mrad}$, the modification of the interference pattern of the HDT at the position of the atoms is negligible. With this method, trapped atoms can be moved in the $x$-direction by up to $40 \mu \mathrm{m}$, i.e., twice the waist radius of the HDT, with a precision of a few micrometers within $50 \mathrm{~ms}$. The storage time of the atoms in the HDT is about $8 \mathrm{~s}$, limited by heating effects caused by the phase noise of the dual-frequency synthesizer driving the AOMs.

In order to actively control the interatomic separations, we use a second, vertical standing wave dipole trap (VDT), operated as optical tweezers, see Fig. 1 The VDT is generated by an Yb:YAG laser beam $\left(\lambda_{\mathrm{VDT}}=\right.$ $1030 \mathrm{~nm}$ ), focused to a waist of $w_{\mathrm{VDT}}=10 \mu \mathrm{m}$ at the position of the HDT. The standing wave is produced by retro-reflecting the beam with a spherical mirror $\mathrm{M}_{3}$. A typical incident power of $0.3 \mathrm{~W}$ results in a measured trap depth of $U_{\mathrm{VDT}} / k_{\mathrm{B}}=1.5 \mathrm{mK}$. The retro-reflecting mirror is mounted on a PZT stage, allowing us to move the standing wave pattern along the VDT axis. We thereby transport atoms in the $z$-direction by typically $60 \mu \mathrm{m}$ with a precision of a few micrometers within $30 \mathrm{~ms}$. The storage time in the VDT is about $13 \mathrm{~s}$ and is limited by heating effects caused by the laser intensity noise of the Yb:YAG-laser. When cooling the atoms with the optical molasses, the storage time in each of the traps can be increased to about $1 \mathrm{~min}$, limited by background gas collisions only.

In both standing wave dipole traps the potential wells are almost two orders of magnitude tighter in the ax-

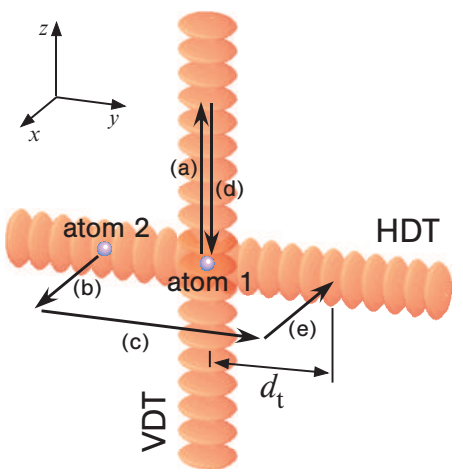

FIG. 2: (Color online) The distance between two atoms which are simultaneously trapped in the horizontally oriented standing wave dipole trap (HDT) can be set to a target distance $d_{\mathrm{t}}$ by our distance-control operation, involving displacements of the atoms in all three spatial dimensions. See text for details.

ial direction than in the radial direction. The maximum axial confining forces are thus much larger than the maximum radial forces. As a consequence, an atom stored in the overlap region of both traps will always follow the axial motion of the traps. This allows us to actively set the distance between the two atoms in the HDT: Atom 1 is first transported along the $y$-direction into the overlap region of both traps. Then, the standing wave pattern of the VDT is axially shifted upwards and atom 1 moves in the positive $z$-direction by about $3 w_{\mathrm{HDT}}$, see Fig. 2(a). At this separation, the HDT exerts negligible forces on atom 1. Atom 2 can now be transported to any position along the HDT with respect to the VDT even when shuttling it through the VDT. By reinserting atom 1 into the HDT, it can hence be placed at any target position relative to atom 2 .

The reinsertion of atom 1 into the HDT is however nontrivial, if the target distance to atom 2 is smaller than the waist of the VDT. In this case, reinserting atom 1 by transporting it along the VDT axis would inevitably expel atom 2 downwards out of the overlap region. We circumvent this problem using the procedure schematically depicted in Fig. 2. The two traps are first horizontally separated by displacing the axis of the HDT in the positive $x$-direction (b). Atom 2 is then transported to the desired $y$-position with respect to the VDT (c), and atom 1 is transported downwards to the vertical $z$-position of the horizontal trap (d). Next, atom 1 is inserted at the desired position by displacing the HDT radially to the $x$-position of the VDT (e). Finally, the VDT is adiabatically switched off. As a result, atom 2 is not expelled out of the overlap region, because no axial motion of the traps is involved when merging them. Therefore, the "radial reinsertion" is compatible with the insertion of atom 1 into the potential well of the HDT already occupied by atom 2 .

We first characterize the performance of our distancecontrol operation for a non-zero target distance of $d_{\mathrm{t}}=$ $15.00 \mu \mathrm{m}$ between the atoms [13]. For this purpose, we 


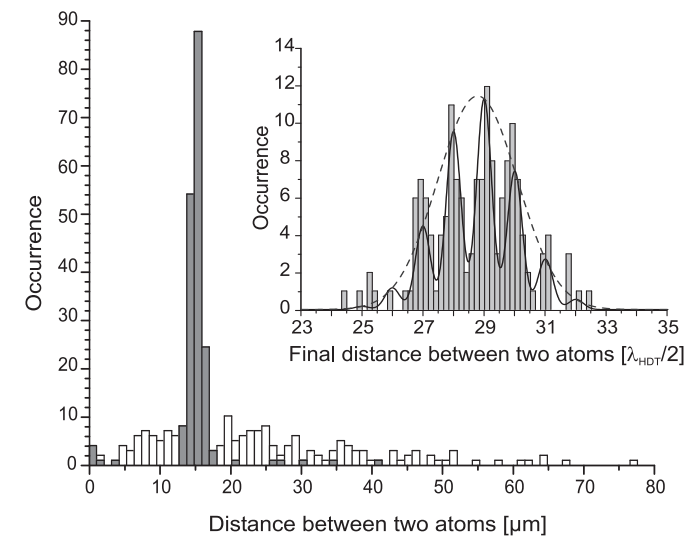

FIG. 3: Performance of our distance-control operation. The white histogram shows the broad distribution of the initial atomic separations for about 190 atom pairs. The grey histogram shows the distribution of final distances for the same pairs after the distance-control operation (target distance $\left.d_{\mathrm{t}}=15 \mu \mathrm{m}\right)$. Inset: Zoom of the distribution of final distances. The histogram clearly shows that the final distances are integer multiples of the standing wave period of $\lambda_{\mathrm{HDT}} / 2$. The solid line is a theoretical fit with a Gaussian envelope (dashed line) centred at $d_{\text {Gauss }}=15.31( \pm 0.07) \mu \mathrm{m}$ and having a $1 / \sqrt{e}$-halfwidth of $\Delta d_{\text {Gauss }}=0.71( \pm 0.05) \mu \mathrm{m}$. The narrow peaks under this envelope have a $1 / \sqrt{e}$-halfwidth of $\Delta d_{\mathrm{ICCD}}=0.130( \pm 0.010) \mu \mathrm{m}$, corresponding to the precision of our distance measurement.

load two atoms on average into the HDT and post-select the events with initially two atoms. We use the radial insertion scheme, as depicted in Fig. 2] with the order of steps (b) and (c) interchanged. The whole procedure, including the initial ICCD image, takes about $2 \mathrm{~s}$, short compared to the trap storage times. The final distance is then checked by recording another ICCD image. Figure 3 shows a histogram (bin size of $\lambda_{\mathrm{HDT}}=$ $1064 \mathrm{~nm}$ ) of the initial distances (white) and final distances (grey) between the atoms for about 190 pairs. Initially, the atoms have random separations of up to $\sim 80 \mu \mathrm{m}$ whereas the final distribution is strongly peaked around $d_{\mathrm{m}}=15.27( \pm 0.05) \mu \mathrm{m}$. Those atom pairs having an initial separation exceeding $10 \mu \mathrm{m}$, i.e., the "size" of the optical tweezers, are rearranged by our distancecontrol operation with a success rate of up to $98_{-5}^{+2} \%$ and a standard deviation of $\Delta d_{\mathrm{m}}=0.78( \pm 0.05) \mu \mathrm{m}[13]$. This spread is mainly due to the precision of the transport of the atoms along the HDT and the accuracy of reinserting atom 1 into the HDT. In previous work we have shown that our transport along the HDT is subject to a statistical error $\Delta y_{\text {transp }}=0.190( \pm 0.025) \mu \mathrm{m} \mathrm{rms}$ [10]. Since the experimental sequence used here involves two transports along the HDT, i.e., moving the atom to be extracted to the $y$-position of the VDT and then placing the remaining atoms at the target distance $d_{\mathrm{t}}$, this effect contributes an uncertainty of $\sqrt{2} \Delta y_{\text {transp }}=$ $0.270( \pm 0.035) \mu \mathrm{m} \mathrm{rms}$ to the final distance between the atoms. Furthermore, immediately after reinserting the extracted atom into the HDT, its measured position has a spread of $\Delta y_{\text {insert }}=0.65( \pm 0.05) \mu \mathrm{m}$ rms [16]. Finally, the distance measurement contributes an uncertainty of $\Delta d_{\mathrm{ICCD}}=0.130( \pm 0.010) \mu \mathrm{m}$. The total expected uncertainty of the measured final distance thus amounts to $\left(2 \Delta y_{\text {transp }}^{2}+\Delta y_{\text {insert }}^{2}+\Delta d_{\text {ICCD }}^{2}\right)^{1 / 2}=0.72( \pm 0.05) \mu \mathrm{m}$.

The fact that the distribution of final distances extends over only a few potential wells of the HDT is strikingly apparent in the inset of Fig. 3. where the histogram of the distribution of final distances is displayed for a smaller bin size of $\lambda_{\mathrm{HDT}} / 12=89 \mathrm{~nm}$. The distribution is clearly peaked with a periodicity of $532 \mathrm{~nm}$, showing that the final distances are integer multiples of the standing wave period $\lambda_{\mathrm{HDT}} / 2$. Given the width of the distribution of true final distances $\Delta d_{\text {true }}=\left(\Delta d_{\text {Gauss }}^{2}-\Delta d_{\mathrm{ICCD}}^{2}\right)^{1 / 2}=$ $0.70( \pm 0.05) \mu \mathrm{m}$, we can estimate the success rate of preparing pairs of atoms separated by a predefined number of potential wells to equal

$$
p_{\text {theor }}=\frac{p_{\text {noloss }}}{\sqrt{2 \pi} \Delta d_{\text {true }}} \int_{-\frac{\lambda_{\mathrm{HDT}}}{4}}^{\frac{\lambda_{\mathrm{HDT}}}{4}} \exp \left(-\frac{y^{2}}{2 \Delta d_{\text {true }}^{2}}\right) d y,
$$

where $p_{\text {noloss }}$ is the probability for not losing an atom during the manipulation. Assuming $p_{\text {noloss }}=1$ for the moment, we obtain $p_{\text {theor }}=30 \pm 2 \%$. In particular, it should be possible to join the two atoms in one and the same potential with a comparable success rate.

The experimental sequence realizing this situation corresponds to the one depicted in Fig. 2 with $d_{\mathrm{t}}$ set to zero. Again, the necessary condition for selectively extracting atom 1 from the HDT is that both atoms be initially separated by more than the $10-\mu \mathrm{m}$ resolution of the optical tweezers. We post-select these events by analysing the initial ICCD fluorescence images. Finally, we discriminate events where atom 1 has successfully been transferred into the potential well containing atom 2 from events where the two atoms occupy different potential wells by inducing two-atom losses. This is achieved by illuminating the atoms with the optical molasses for $1 \mathrm{~s}$. It has been shown that radiative escape is the leading physical mechanism for light induced collisions under these conditions [17]. The resulting energy release causes both atoms to leave the trap in most cases. If, on the other hand, the atoms reside in different potential wells, radiative escape is not possible and the atoms remain trapped. Detecting the absence of the pair of atoms after the optical molasses stage therefore confirms the successful joining of the two atoms in one potential well of the HDT.

In order to independently examine the dynamics of this collisional process, we load a variable number of atoms from the MOT into the HDT, illuminate them with the optical molasses, and detect the atomic fluorescence with the APD. The level of this fluorescence signal is a measure of the number of trapped atoms. If we load on average 3 atoms per shot distributed over about 25 potential wells into the HDT, the probability for having two atoms in one potential well is negligibly small. In this case, their fluorescence level remains constant, i.e., no atom losses 


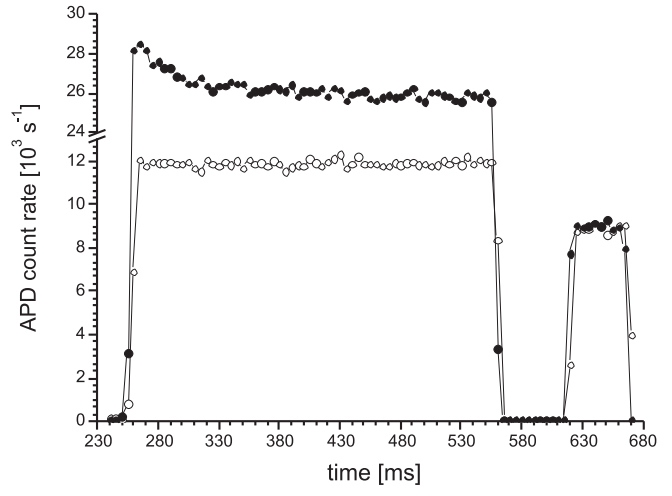

FIG. 4: Fluorescence signal of on average 3 atoms (open circles) and on average 19 atoms (full circles) trapped in about 25 potential wells of the HDT. At $t=260 \mathrm{~ms}$ the optical molasses illuminating the atoms is switched on. At $t=560 \mathrm{~ms}$, the remaining atoms are ejected from the HDT by switching off all lasers for $50 \mathrm{~ms}$ in order to measure the background signal due to stray light. Each of the two traces is averaged over 100 shots. See text for details.

are detected, see open circles in Fig. 4. For on average 19 atoms per shot distributed over about 25 potential wells, however, the probability for at least two atoms occupying a common well is significant. In this case, we observe an exponential decay of the average fluorescence level to a steady state value within about $150 \mathrm{~ms}$, i.e., the optical molasses results in radiative escape of atoms within a few hundred milliseconds, see filled circles in Fig. 4 The steady state fluorescence level then corresponds to the atoms which are trapped in individual potential wells of the HDT. Our choice of $1 \mathrm{~s}$ illumination time thus ensures that all pairs of atoms undergo a light induced collision.

Since we cannot distinguish a two-atom loss due to radiative escape from two uncorrelated one-atom losses during the experimental sequence, we need to quantify the latter in an independent measurement. For this purpose, we have carried out the entire experimental sequence with only atom 1 present and only atom 2 present.
In both cases, we have measured the loss probability $p_{i}$ of atom $i$, yielding $p_{1}=6.5_{-2.4}^{+2.1} \%$ and $p_{2}=0.0_{-0.0}^{+3.5} \%$, respectively. From these measurements, we infer the probability for two uncorrelated one-atom losses during the experimental sequence to be $p_{\text {uncorr }}=p_{1} \cdot p_{2}=$ $0.0_{-0.0}^{+0.2} \%$. Furthermore, the probability for not losing any of the two atoms during the manipulation is $p_{\text {noloss }}=\left(1-p_{1}\right)\left(1-p_{2}\right)=94_{-5}^{+6} \%$. In the present measurement, $\Delta y_{\text {insert }}=0.82( \pm 0.11) \mu \mathrm{m} \mathrm{rms}$, yielding $\Delta d_{\text {true }}=0.86( \pm 0.11) \mu \mathrm{m}$. According to Eq. (1), the probability for successfully inserting atom 1 into the potential well of atom 2 should thus ideally be $p_{\text {theor }}=$ $23_{-3}^{+3} \%$

Carrying out the experimental sequence with both atom 1 and atom 2, we measure a total two-atom loss probability of $p_{\text {meas }}=16_{-3}^{+4} \%$. Compared with this value, the probability of uncorrelated two-atom losses $p_{\text {uncorr }}$ is negligible, proving the successful joining of the two atoms in one potential well. Note that, in former work, we have found experimental evidence that lightinduced collisions can also lead to one-atom losses 18]. Taking this effect into consideration, the true success rate might then even be higher than $p_{\text {meas }}$.

Summarizing, we have inserted two atoms into a single potential well of a standing wave optical dipole trap and we have deterministically induced interactions between these atoms leading to light-induced collisions. The presented results open the route towards fascinating experiments. In particular, using photoassociation techniques, it should become possible to build a single ultracold diatomic molecule from its constituents and to store and to manipulate this molecule inside our standing wave dipole trap. Furthermore, by exploiting coherent spin-changing collisions between two atoms trapped inside the same potential well, one might be able to prepare an entangled Bell pair of atoms which could then be used as a resource for quantum information processing schemes.

We acknowledge valuable discussions with M. Karski. This work was supported by the DFG and the EC. I. D. acknowledges funding by INTAS.
[1] T. Rom et al., Phys. Rev. Lett. 93, 073002 (2004).

[2] K. Xu et al., Phys. Rev. A 72, 043604 (2005).

[3] C. Ryu et al., cond-mat/0508201

[4] T. Stöferle, H. Moritz, K. Günter, M. Köhl, and T. Esslinger, Phys. Rev. Lett. 96, 030401 (2006).

[5] G. Thalhammer et al., Phys. Rev. Lett. 96, 050402 (2006).

[6] T. Volz et al., cond-mat/0605184

[7] O. Mandel et al., Nature (London) 425, 937 (2003)

[8] A. Widera et al., Phys. Rev. Lett. 95, 190405 (2005).

[9] I. Bloch, Nature Physics 1, 23 (2005) and references therein.

[10] I. Dotsenko et al., Phys. Rev. Lett. 95, 033002 (2005).

[11] D. Schrader et al., Phys. Rev. Lett. 93, 150501 (2004).

[12] S. Kuhr et al., Science 293, 278 (2001).

[13] Y. Miroshnychenko et al., Nature (London) in press
(2006).

[14] Y. Miroshnychenko et al., Opt. Express 11, 3498 (2003).

[15] D. Schrader et al., Appl. Phys. B 73, 819 (2001).

[16] The main contributions to this value are the fluctuations and drifts of the $y$-position of the VDT of $0.5( \pm 0.2) \mu \mathrm{m}$ rms (measured by observing the $y$-position of atoms trapped in the VDT with the ICCD camera). A further contribution to $\Delta y_{\text {insert }}$ comes from the finite confinement of atom 1 in the VDT due to its thermal motion along the $y$-direction.

[17] S. J. M. Kuppens, K. L. Corwin, K. W. Miller, T. E. Chupp, and C. E. Wieman, Phys. Rev. A 62, 013406 (2000).

[18] B. Ueberholz, S. Kuhr, D. Frese, V. Gomer, and D. Meschede, J. Phys. B: At. Mol. Opt. Phys. 35, 4899 (2002). 